\title{
LA CONTRATACIÓN DE TRABAJADORES A TRAVÉS DE EMPRESAS DE SERVICIOS TEMPORALES
}

\author{
GERARDO ARENAS MONSALVE
}

This article analyzes Temporary Agencies (Empresas de Servicios Temporales) in the context of work flexibility, and it also analyzes the evolution of norms that regulate this field in Colombia. The article then studies the country's Temp Agencies laws and regulation. Finally, it examines how judges from the Colombian Supreme Court and Constitutional Court have treated the subject, highlighting the legal consequences of overstepping the normative limits of this activity.

\section{El contexto general: la flexibilización laboral}

El tema de la contratación de trabajadores a través de las Empresas de Servicios Temporales (en adelante, EST) no es un aspecto técnico-jurídico aislado, sino que debe ser examinado en el contexto más amplio de la flexibilización laboral que conoce hoy el mundo de las relaciones laborales.

Por qué surge la flexibilización laboral

Como lo ha señalado un importante estudio de Arturo Bronstein, funcionario de la O.I.T., en los debates sobre la flexibilidad laboral se encuentran factores económicos y tecnológicos, factores ideológicos y factores culturales ${ }^{1}$ :

Los factores económicos y tecnológicos sitúan las raíces de los planteamientos flexibilizadores en los cambios que se dieron en Europa a partir de los años sesenta, al pasar de un importante crecimiento económico que venía desde la finalización de la segunda guerra mundial, a un período de reestructuracio-

\footnotetext{
${ }^{1}$ Bronstein, Arturo: «La flexibilidad del trabajo: Panorama general», en La flexibilización del trabajo. Un estudio internacional. Coordinador Oscar Hernández Alvarez. Instituto Latinoamericano de Derecho del Trabajo, Barquisimeto, 1990, pp. 13 y ss.
} 
nes industriales, irrupción de nuevas tecnologías, el surgimiento de nuevas potencias industriales (cuenca del Pacífico) y la crisis energética.

Los factores ideológicos están enmarcados en el cuestionamiento al Estado de bienestar y el surgimiento de una mentalidad política con mayor énfasis en el mercado como mecanismo de asignación de recursos en la sociedad. En ese contexto «eran las fuerzas del mercado y no el gobierno a través de su política de regulación institucional, quienes deberían regular la economía, incluyendo al propio mercado de trabajo». La protección laboral pasó a ser vista, entonces, «como un elemento negativo que imponía rigideces», por lo que se pasó a revisar la protección social y laboral existente ${ }^{2}$.

Los factores culturales que determinaron el surgimiento de la flexibilización son los que Bronstein denomina las «presiones psicosociales provenientes de los propios trabajadores» y que describe así:

El derecho del trabajo tradicional (...) se construyó en torno de un modelo de relación de trabajo cuyo arquetipo era el empleo industrial, desempeñado por un trabajador manual, de sexo masculino, en un régimen de jornada completa, quien entraba por lo común muy joven en el mercado de trabajo.

En las últimas décadas, ese perfil cambió sustancialmente, y los trabajadores y trabajadoras de hoy no encajan en ese modelo tradicional, por razones que sobra explicar.

\section{Las formas jurídicas que asume la flexibilidad}

El estudio que se viene citando señala que hay en las distintas legislaciones nacionales cuatro grandes vertientes de la flexibilidad laboral: la flexibilidad de los salarios, la flexibilidad de la duración del trabajo, la flexibilidad de la organización del trabajo y la flexibilidad de la contratación laboral ${ }^{3}$.

La flexibilidad de los salarios se planteó en algunos países a partir de la idea de que los mismos se venían fijando por mecanismos demasiado rígidos (decretos de salarios mínimos generales, convenios colectivos que establecen mínimos profesionales en niveles muy altos o mecanismos de indexación automática). También se plantearon mecanismos de reducción convencional de salarios por causa de la crisis.

\footnotetext{
${ }^{2}$ Ibídem, p. 20.

${ }^{3}$ Ibídem, p. 24 y ss.
} 
La flexibilidad en la duración del trabajo parte del planteamiento de las dificultades que enfrentan las empresas cuando procuran adaptar las horas del trabajo al nivel requerido por la coyuntura. A este respecto, surgieron mecanismos de reducción y ampliación de horarios de trabajo según las circunstancias productivas, así como el establecimiento de horarios flexibles.

La flexibilidad en la organización del trabajo ha tenido menos importancia relativa desde el ángulo jurídico que las demás formas de flexibilidad. Está relacionada con la rigidez en las clasificaciones de puestos de trabajo y el debate acerca de si el trabajador tiene derecho adquirido a una calificación profesional determinada.

La flexibilidad en la contratación laboral ha sido tal vez el elemento de mayor impacto entre los planteamientos flexibilizadores. A este respecto, se han planteado tres formas principales de flexibilización: la primera es la que se refiere al monopolio en el reclutamiento de trabajadores, en los países que lo tenían en manos del servicio público de empleo. La segunda alude a la flexibilidad en las formas de terminación del vínculo laboral y la disminución de la estabilidad laboral que tradicionalmente pregonaba el Derecho Laboral. Y la tercera se relaciona con las modalidades específicas en que se presta el trabajo, las que dieron lugar a las llamadas «relaciones de trabajo atípicas»: trabajo a tiempo parcial, trabajo intermitente, contratos de duración determinada, programas de empleo formación, «teletrabajo», trabajo mediante vínculo autónomo, etc. En este grupo de aspectos de la flexibilidad en la contratación se sitúan precisamente las relaciones de trabajo «triangulares», y entre ellas la intermediación de las agencias de trabajo temporal.

Estas tendencias flexibilizadoras de la contratación laboral, con sus consecuencias específicas en la legislación del trabajo, no parecen tampoco ser definitivas. Medidas más recientes en algunos países europeos -España, por ejemplo- muestran un cuestionamiento de la flexibilización o por lo menos su razonable atenuación. El profesor Montoya Melgar se ha expresado así sobre el particular:

Tras la euforia precarizadora y después de años de proclamación del fin del principio de estabilidad en el empleo y de encomio de los benéficos efectos de la contratación temporal sobre el mercado de trabajo, los tiempos más próximos nos muestran otros efectos menos deseados; por citar sólo los económicos, se comprueba como la expansión de los contratos temporales desemboca en la contracción del consumo y consiguientemente de la producción, en la escasa cualificación y experiencia de la mano de obra, en la obstaculización de la introducción de nuevas tecnologías, en la pérdida de eficacia del sistema productivo al 
estar basado éste cada vez más en trabajos no estables, en el incremento de la siniestralidad laboral, etc..

En ese contexto se introdujo la reforma española de $1994^{4}$

\section{El auge del trabajo temporal}

Aparecidas en los Estados Unidos a partir de la segunda post-guerra, las empresas de trabajo temporal vinieron a satisfacer una necesidad de la vida empresarial moderna: la de resolver, en forma rápida y eficiente, las necesidades ocasionales de personal, mediante el envío de trabajadores que dependían de la empresa temporal y no ocasionaban responsabilidades laborales a la empresa que utilizaba el servicio.

La política normativa de los distintos países sobre el nuevo fenómeno fue variada y puede clasificarse así: Un primer grupo de países decide adoptar una política de rigurosa prohibición, por considerar que practicaban actividades de colocación y restringían derechos laborales de los trabajadores. Un segundo grupo de países adopta una postura liberal de no intervención legislativa sobre estas empresas. Finalmente, los que adoptaron la posición intermedia de no prohibir la actividad, por considerarla necesaria en una economía libre, pero deciden introducir regulaciones normativas para introducir límites a su actividad.

Con el surgimiento de las políticas flexibilizadoras en las relaciones laborales que se han descrito antes, las contrataciones de trabajadores a través de empresas temporales tuvieron un incremento significativo y menores restricciones en la legislación.

En los últimos años, como lo señala Montoya Melgar,

la valoración sobre la experiencia de funcionamiento de esas empresas de trabajo temporal no es unánime: mientras que de un lado se destaca su valor de generación de empleo, de especialización de los servicios y de polivalencia de formación, desde el ángulo opuesto se denuncian los problemas de precarización laboral que originan ${ }^{5}$.

\footnotetext{
${ }^{4}$ Montoya Melgar, Alfredo, «Estabilidad en el empleo: cara y cruz de un principio», en Derecho Social No. 50, Colegio de Abogados del Trabajo, Bogotá, marzo de 1999, pp. 1 y ss.

${ }^{5}$ Ibídem, p. 5.
} 


\section{Las políticas normativas sobre el trabajo temporal en Colombia}

Acerca de la actividad de las EST en Colombia, presentes en la economía colombiana desde hace cerca de cuarenta años, el Estado colombiano registra tres etapas en su política normativa frente a las mismas: una primera etapa, que podríamos llamar de abstención regulativa; una segunda etapa que sería de «regulación permisiva» y un último período de regulación y control formal. Explicaremos brevemente esta periodización.

\section{Primera etapa: abstención normativa (1963-1983)}

Según lo registran los pocos análisis que se han hecho sobre el tema en el país, en 1963 hizo su entrada en Colombia la primera empresa de servicio temporal: la multinacional «Manpower», que operó hasta 1971, cuando se desarticuló y se fraccionó en varias EST con capital nacional ${ }^{6}$.

La característica básica de este primer período está en que el funcionamiento de las EST careció de regulación normativa específica. Pese a que esa carencia podía favorecer su funcionamiento, en cuanto les permitía fijar sus propias reglas de juego, el asunto resultaba más complicado, por cuanto las EST eran vistas como un «apéndice» de las agencias de colocación de empleo, que en su tiempo estaban reguladas por un decreto del gobierno (Decreto 2676/71), y cuya actividad estaba controlada por Convenios de la OIT. El propio gremio de las empresas temporales -ACOSET- inició un plan de propuesta legislativa tendiente a encontrar una regulación autónoma para las EST.

\section{Segunda etapa: regulación permisiva (1983-1991)}

Dentro de las iniciativas para regular la actividad de las EST, el Gobierno presentó al Congreso proyectos de ley sobre el tema, hasta que en la administración del Presidente Belisario Betancur el Gobierno llegó a la conclusión de que la regulación de las EST podía efectuarse por decreto. En la elaboración del proyecto tuvo una notable injerencia el propio gremio de la actividad, es decir, ACOSET?

Se expidió entonces el Decreto 1433 de 1983, que reconoce a las EST como empleadores de sus trabajadores y no como intermediarios. El decreto establecía los requisitos de constitución de las EST, y asignaba al Ministerio de Trabajo

\footnotetext{
${ }^{6}$ Duque Villegas, Francisco Javier y Arana Madriñán, Luis Felipe, "Las empresas de servicio temporal. Su problemática a la luz de las actuales realidades -Un examen crítico”-. Tesis de grado, Pontificia Universidad Javeriana, Bogotá, 1989, pp. 10 y ss.

${ }^{7}$ Ibídem, pp. 33-54.
} 
la vigilancia y control de su actividad. El mayor defecto de esta reglamentación consiste en que no se establece término alguno a la «temporalidad» del servicio suministrado por la EST.

La regulación del Decreto 1433/83 dejó satisfechas a las EST, que podían ofrecer a sus usuarios la condición de «verdaderos patronos» de los trabajadores temporales contratados, pero que podían mantener indefinidamente a estos trabajadores, eludiendo así responsabilidades extralegales.

Desde el punto de vista jurídico, la legalidad de esta regulación fue cuestionada: algunos analistas consideraron que no era posible considerar, por vía de decreto reglamentario, que las EST fueran verdaderos patronos, cuando frente al marco legal general (CST, art. 34 y 35) constituían una modalidad del «simple intermediario» ${ }^{8}$.

$\mathrm{Al}$ amparo de ese marco normativo permisivo y favorable a sus intereses, las EST continuaron su crecimiento en el mercado laboral colombiano.

\section{Tercera etapa: regulación y control formal (desde 1991)}

Con los debates de la reforma laboral que culminó en la Ley 50 de 1990 se introdujeron en la legislación laboral colombiana importantes mecanismos de flexibilidad laboral. Entre ellos, se facilitó que los empleadores contrataran directamente trabajadores por períodos de corta duración (contratos de trabajo a término fijo inferior a un año), situación que la legislación anterior dificultaba, lo que a su vez estimulaba la contratación a través de las EST. En ese contexto, la reforma laboral se propuso crear un nuevo marco normativo para las mismas.

En la Exposición de Motivos del Proyecto de Ley, el Ministro de Trabajo de la época señalaba la importancia de legislar sobre el asunto «con el objeto de 212 que esta actividad tenga un marco legal adecuado y se protejan debidamente los derechos de los trabajadores». Destaca que «la temporalidad es de la esencia de tales contratos», así como otros aspectos del proyecto: que los contratos consten por escrito, que haya pólizas para garantizar las obligaciones de las EST con los trabajadores en misión, y que el Ministerio verifica sus requisitos para operar y que debe controlar permanentemente su actividad'.

En la ponencia para primer debate se señala que «el control de las agencias temporales de empleo es un tema clave para que la reforma laboral cumpla el objetivo de mejorar la estabilidad de los trabajadores colombia-

\footnotetext{
${ }^{8}$ Ochoa Moreno, Benjamín, «Los intermediarios o la sobreexplotación de la mano de obra asalariada», Revista Trabajo y Derecho, No. 12-13, Bogotá, Octubre de 1984, pp. 27 y ss.

${ }^{9}$ Ministerio de Trabajo y Seguridad Social, Compilación de la Reforma Laboral, Imprenta Nacional, Bogotá, 1991, p. 69.
} 
nos». Se introduce la limitación a un año, con prórroga, el período máximo de contratación, que el proyecto original del gobierno había señalado en dos años prorrogables; también el respeto al principio de igualdad salarial y finalmente el derecho proporcional del trabajador en misión a vacaciones y prima de servicios ${ }^{10}$.

La ponencia para segundo debate insiste en que se requiere «una reglamentación tendiente a la eliminación de abusos que pueden cometerse en detrimento de los trabajadores». Señala que «se consideró necesario restringir aún más el campo de acción de estas empresas» y que «se establecen normas muy precisas tendientes a la protección de los derechos de los trabajadores temporales en cuanto a su remuneración, prestaciones sociales y salud ocupacional» ${ }^{11}$.

Si se observan en detalle las normas del proyecto original del gobierno y las modificaciones que se fueron introduciendo en el debate parlamentario, es posible sacar dos conclusiones importantes: la primera es que el proyecto original del gobierno era muy laxo en la regulación de las EST, al punto que la regulación no difería sustancialmente de la anterior. La segunda conclusión es que pese a los mayores controles y exigencias que el debate parlamentario logró introducir, de todas maneras, la regulación de las EST, tal como quedó establecida en la Ley 50 de 1990 contiene serias deficiencias, como se examinará en detalle más adelante.

\section{El marco legal vigente de las empresas de servicios temporales}

\section{Nociones legales}

Las EST constituyen una modalidad de trabajo en la que no existe vínculo directo entre quien se beneficia del trabajo y la persona que lo realiza, sino que se trata de un vínculo laboral indirecto: en efecto, existe una vinculación laboral, que supone formalmente todos los derechos para el trabajador, pero ese vínculo lo tiene el trabajador con una empresa especializada en esa actividad y bajo un marco legal específico.

El marco legal actual de las EST está contenido en la Ley 50/90 (arts. 71 a 94) y el Decreto 24/98, modificado por el Decreto 503/98. De conformidad con la ley:

es empresa de servicios temporales aquella que contrata la prestación de servicios con terceros beneficiarios para colaborar tem-

${ }^{10}$ Ibídem, p. 130.

${ }^{11}$ Ibídem, pp. 167-168. 
poralmente en el desarrollo de sus actividades, mediante la labor prestada por personas naturales, contratadas directamente por la empresa de servicios temporales, la cual tiene con respecto de éstas el carácter de empleador (art. 71)

Los trabajadores de las EST son de dos clases: trabajadores de planta, que desarrollan su actividad en las dependencias de las EST; y trabajadores en misión, que son «aquellos que la empresa de servicios temporales envía a las dependencias de sus usuarios a cumplir la tarea o servicio contratado por éstos» (art. 74).

La ley fija las reglas de constitución de las EST, como la de constituirse como persona jurídica y tener como objeto exclusivo esa actividad (art. 72); exige la autorización del Ministerio de Trabajo (art. 82), para lo cual deben cumplir determinados requisitos, entre los que figura la obligación de constituir una garantía para asegurar los derechos laborales de los trabajadores (art. 83); y deben tener un reglamento interno de trabajo con cláusulas especiales (art. 85).

Tipos de vínculos jurídicos y sus reglas legales

En la regulación de las EST es preciso ver, en consecuencia, una relación triangular, en la que hay tres tipos de vínculos jurídicos:

- Entre la EST y la empresa usuaria, hay un contrato de prestación de servicios. Ese contrato debe constar por escrito y tiene reglas específicas sobre las obligaciones laborales que asume la EST (art. 81). La ley prohibe a las EST prestar servicios a usuarias con las cuales tengan vinculación económica (art. 80), como también a usuarias en huelga (art. 89). La limitación más importante es la que señala cuándo los usuarios pueden contratar con las EST: en labores ocasionales, por reemplazos de personal o para atender incrementos de la actividad respectiva de la usuaria «por un término de seis (6) meses prorrogables hasta por seis (6) meses más» (art. 77).

- Entre la EST y el trabajador hay un contrato de trabajo. Ese contrato somete a los trabajadores en misión de la EST a toda la normatividad laboral del Código Sustantivo del Trabajo (art. 75), con ligeras variaciones respecto del pago de vacaciones y prima (art. 76). Y se ordena que los trabajadores en misión «tendrán derecho a un salario ordinario equivalente al de los trabajadores de la empresa usuaria que desempeñen la misma actividad», así como los beneficios que la usuaria tenga en materia de transporte, alimentación y recreación (art. 79).

- Entre la empresa usuaria y el trabajador hay un vínculo que no constituye contrato pero origina algunos derechos. En la regulación legal de las EST es 
claro que entre la empresa usuaria y el trabajador temporal no hay contrato alguno, pues el empleador es la EST, y ésta ha celebrado un contrato de servicios con la usuaria. Pero de hecho, el trabajador labora en las dependencias, en las condiciones y bajo las órdenes de la empresa usuaria. Ese vínculo, entonces, genera derechos para el trabajador en misión, tales como el de igualdad salarial con los trabajadores de la usuaria, así como el derecho a gozar de los beneficios de transporte, alimentación y recreación de la usuaria. Además, de conformidad con las normas reglamentarias del sistema de riesgos profesionales, «las empresas usuarias deberán incluir a los trabajadores en misión dentro de sus Programas de Salud Ocupacional», debiendo suministrarles inducción, información permanente sobre riesgos, elementos de protección personal y condiciones de seguridad, higiene y medicina laboral que la usuaria tenga para sus trabajadores; además, la EST debe cotizar a riesgos profesionales sobre la clase de riesgo en que se encuentra clasificada la empresa usuaria (D. 1530/96, art. 11 a 13).

La Ley 50/90, excluyó expresamente de esta regulación de las EST a las empresas que prestan «servicios diferentes al envío de trabajadores en misión», y señaló específicamente «las de suministro de alimentación y las que realizan labores de aseo». Esta exclusión deja a esas actividades de intermediación sin un marco legal específico, de modo que pueden ser, conforme al Código Laboral unos «contratistas independientes», o unos «simples intermediarios» o constituir modalidades de contratos de servicios donde puede discutirse si existe o no contrato de trabajo con la empresa que se beneficia de su actividad.

\section{Reglamentaciones}

Las normas de la Ley 50 de 1990 sobre las EST han sido objeto de dos reglamentaciones. La primera fue el Decreto 1707 de 1991 y la segunda fue proferida mediante el Decreto 24 de 1998, que derogó expresamente el anterior.

La reglamentación vigente se ocupa principalmente de los siguientes asuntos: señala que la EST tendrá siempre el carácter de empleador de los trabajadores de planta y de los trabajadores en misión (art. $1^{\circ}$ ). Dispone también que la seguridad social (en cuanto a salud, pensiones y riesgos profesionales) es responsabilidad de la EST, pero sin perjuicio de las responsabilidades que la ley asigne a las empresas usuarias en estas materias (art. $4^{\circ}$ ). Reglamenta al detalle los documentos que deben aportarse para que el Ministerio de Trabajo pueda extender la autorización de funcionamiento de la EST (art. $7^{\circ}$ ), así como las obligaciones de éstas frente al Ministerio: actualización de cuantías de pólizas, informes estadísticos, reformas estatutarias, constitución de sucursales o agencias, etc. (Art. $8^{\circ}$ a 12 ). 
Un aspecto importante de la reglamentación, que no es original del Decreto 24/98 sino adicionado por el Decreto 503/98, consiste en la precisión que se introduce acerca de la duración que puede tener el vínculo del trabajador temporal, cuando se trate de incrementos de producción, transporte, ventas o prestación de servicios: la ley establece que en esos eventos la contratación de temporales sólo puede hacerse por un término de seis meses prorrogables por seis meses más (Ley 50/90, art. 77; D.R. 24/98, art. 13). El Decreto 503/98 agregó un parágrafo que dispone que si se ha cumplido el plazo de seis meses y su prórroga, y la necesidad en la usuaria subsiste, «para atender esa necesidad la empresa no podrá contratar con empresas de servicios temporales». Esta prohibición, evidentemente, busca que no se desconozcan los plazos máximos de vinculación, lo que sigue siendo uno de los problemas más serios de la actividad de las EST.

Finalmente, la reglamentación se ocupa del régimen sancionatorio de las EST. Comprende multas hasta de 100 salarios mínimos mensuales (art. 19), suspensión de la autorización de funcionamiento (art. 20) y cancelación de la autorización de funcionamiento (art. 21).

Con respecto a la reglamentación del régimen de las EST en la Ley 50/90 se ha pronunciado el Consejo de Estado. Mediante Auto del 28 de mayo de 1998, suspendió provisionalmente una norma del Decreto 24/98 que daba a los trabajadores en misión «todas aquellas prerrogativas que la empresa usuaria tenga respecto de sus propios trabajadores» (art. $3^{\circ}$, literal b), por exceder el marco legal de la Ley 50/ 90 que sólo las obliga a otorgar los beneficios que tenga la empresa usuaria en materia de transporte, alimentación y recreación ${ }^{12}$.

Las empresas de servicios temporales en la jurisprudencia de la Corte Suprema de Justicia

Desde la regulación anterior a la Ley 50/90, la Corte Suprema de Justicia ha precisado que una característica básica de las EST la constituye el ser «verdadero empleador» de los trabajadores en misión. Así lo señaló en sentencia de la Sala de Casación Laboral del 29 de octubre de $1986^{13}$.

Ya en vigencia de la regulación actual, la Sala Laboral de la Corte Suprema de Justicia, en sentencia de casación del 24 de abril de 1997, con ponencia del Magistrado Francisco Escobar Henríquez, ha reiterado el carácter de empleador de la EST, señalando que la empresa usuaria ejerce la potestad de subordinación frente a los trabajadores en misión, pero no por derecho propio sino en virtud de delegación o representación de la EST ${ }^{14}$.

\footnotetext{
${ }^{12}$ Ver Revista Jurisprudencia y Doctrina, Legis, Bogotá, 1998, p. 1409.

${ }_{13}^{13}$ Ver Revista Jurisprudencia y Doctrina, Legis, Bogotá, 1986, p. 1003.

${ }^{14}$ Ver Revista Jurisprudencia y Doctrina, Legis, Bogotá, 1997, p. 683.
} 
En este fallo, la Corte Suprema elabora su más completa jurisprudencia sobre las EST y señala que

los usuarios no responden por los salarios, prestaciones e indemnizaciones de los trabajadores en misión ni de su salud ocupacional, aunque en este aspecto pueden contraer obligaciones con la EST. La razón de que el usuario no deba responder por los derechos laborales de los trabajadores de la EST la explica así la Corte: «Acontece que precisamente mediante el contrato con la EST y con autorización legal, el usuario cancela un sobrecosto sobre el valor real de la fuerza de trabajo que requiere para su actividad económica, a fin de hacerse irresponsable en lo que hace a la remuneración, prestaciones y derechos de los operarios. Desde luego, no se desconoce que por esta razón, entre otras, se ha cuestionado seriamente la institución, con argumentos cuya razonabilidad corresponde estudiar al legislador, mas ello no le resta validez jurídica a los preceptos que en la actualidad permiten y regulan su funcionamiento».

No obstante lo anterior, para la Corte, la ausencia de responsabilidad laboral de la empresa usuaria frente al trabajador temporal admite dos excepciones:

La primera excepción a la no responsabilidad de la usuaria frente al trabajador se da cuando la EST se sale del marco legal establecido:

Pero esta irresponsabilidad laboral del usuario con referencia a los trabajadores en misión - dice la Corte- supone que la EST funcione lícitamente... pues de lo contrario la EST irregular sólo podría catalogarse como un empleador aparente y un verdadero intermediario que oculta su calidad, en los términos del artículo 35-2 del CST», de forma que el usuario ficticio se consideraría verdadero patrono y la supuesta EST pasaría a responder solidariamente de las obligaciones laborales conforme al ordinal $3^{\circ}$ del citado artículo del CST. Igualmente, aparte de las sanciones administrativas que procedan, el usuario se haría responsable en la forma que acaba de precisarse con solidaridad de la EST, en el evento de que efectúe una contratación fraudulenta, vale decir, transgrediendo los objetivos y limitaciones fijados por el artículo 77 de la Ley 50 de 1990, bien sea en forma expresa o mediante simulación. Ello por cuanto las normas que regulan el trabajo humano son de orden público, de obligatorio acatamiento y la ilegalidad o ilicitud se sanciona con la ineficacia de las respectivas estipulaciones (CST, art. 14 y 43). 
Lo expresado en el conciso lenguaje jurídico de la jurisprudencia significa, en la práctica, algo de suma trascendencia que no suelen conocer las empresas usuarias de las EST: si una EST opera sin el permiso del Ministerio de Trabajo (lo que no es frecuente), o si una empresa usuaria contrata trabajadores para labores distintas de las ocasionales, de reemplazo de personal en vacaciones, licencia o incapacidad, o si la vinculación del trabajador temporal excede el término máximo de un año (lo que sucede cotidianamente), la EST deja de ser «verdadero empleador» y pasa a ser considerada «un simple intermediario que oculta su calidad». Es decir, que el empleador, para todos los efectos laborales, será la empresa usuaria; la EST, al pasar de empleador a «simple intermediario», se vuelve solidariamente responsable de esas obligaciones laborales.

El segundo caso en que la empresa usuaria puede resultar responsable como empleador frente al trabajador en misión, conforme a la jurisprudencia que se viene estudiando ocurre cuando la EST acuerda con el trabajador «actividades paralelas ajenas totalmente a las propias del encargo a que se comprometió la EST». A este respecto, la Corte se remite a su propio precedente del 12 de marzo de 1997, con ponencia del Magistrado José Roberto Herrera Vergara ${ }^{15}$. Se trataba del caso de una empresa industrial que contrató los servicios de una EST, la cual vinculó a un trabajador para realizar las labores propias de operario de la empresa industrial; el trabajador fue encargado por la usuaria de una labor de arreglo de tejados, en desarrollo de la cual fue víctima de accidente grave. La Corte consideró que hubo culpa patronal directa de la empresa usuaria, con esta consideración jurídica:

Estima la Sala, en consecuencia con lo dicho, que si bien en principio las empresas de servicios temporales son verdaderos patronos y responsables frente al trabajador en misión de la salud ocupacional, no es lícito ni legítimo que un usuario aproveche los servicios de esta clase de trabajadores para atribuirles funciones que escapan totalmente de los deberes propios del contrato de trabajo celebrado por el empleado con la empresa de servicios temporales y luego pretenda desconocer las naturales consecuencias del marco obligacional que surge del contrato de prestación de servicios celebrado con ésta, para así evadirse de la ineludible responsabilidad laboral que surge de su proceder culposo que origina accidentes de trabajo, los cuales deben ser reparados en la forma prescrita por el artículo 216 del Código de Trabajo.

\footnotetext{
${ }^{15}$ Revista Jurisprudencia y Doctrina, Legis, Bogotá, 1997, p 507.
} 


\section{Y agrega la Corte:}

Y si aparece diáfano -como en el presente caso- que la empresa de servicios temporales fue totalmente ajena a esa actuación apartada del objeto del contrato de prestación de servicios con la usuaria y del contrato de trabajo en misión, ella no puede reputarse subordinante en estos eventos, y por lo tanto no será ésta quien deba satisfacer las indemnizaciones pertinentes sino el empresario usuario culpable de la acción o la omisión generadora del infortunio laboral».

Concluye la Corte para el caso en estudio que, dado que el accidente de trabajo del trabajador en misión «no tuvo como causa el contrato de trabajo con la empresa de servicios temporales, ni fue responsable ésta de su ocurrencia, ni estaba en sus manos poder evitarlo», el único responsable es el usuario, quien incurrió en culpa por haber ordenado al trabajador «por fuera del contrato de trabajo en misión, un trabajo riesgoso a un servidor no capacitado para la labor».

Se concluye, entonces, a la luz de la normatividad y de la jurisprudencia de la Corte Suprema, que en principio las EST son verdaderos empleadores y que las empresas usuarias no tienen obligaciones laborales frente al trabajador en misión. Pero esa regla no es absoluta, pues la jurisprudencia laboral ha señalado las situaciones en las cuales la empresa usuaria asume la condición de empleador frente al trabajador en misión.

\section{Las empresas de servicios temporales en la jurisprudencia de la Corte Constitucional}

La Corte Constitucional ha tenido escasas oportunidades de examinar la constitucionalidad del marco legal de las EST en la Ley 50 de 1990. La única decisión de fondo sobre esa normatividad ha sido la sentencia C-330/ 95, con ponencia del Magistrado Jorge Arango Mejía , en la cual la Corte declaró ajustado a la Constitución el artículo 77 de la Ley 50/90 que regula las ocasiones en las cuales es posible que las EST contraten trabajadores en misión, es decir, para trabajos ocasionales o transitorios, para el reemplazo o incremento ocasional de personal y en cualquier otro caso de necesidad temporal se permite la contratación máxima de temporales por un año (seis meses prorrogables por otro tanto). La demandante argumentaba que las EST eran organizaciones empresariales como cualesquiera otras, de modo que «la limitación de su capacidad de contratación con sus clientes no tiene 
justificación ni explicación», por lo cual estimaba que la norma violaba la libertad de empresa y el principio de igualdad constitucional. La Corte consideró que no existía tal violación de las normas constitucionales señaladas, por cuanto «es claro que la finalidad de la norma es la protección de los trabajadores para que las empresas no abusen de la posibilidad de contratar trabajadores temporales haciendo a un lado los permanentes». La Corte consideró, además, que los tres casos en que se permite la contratación son «razonables», y en especial la limitación temporal del vínculo, que la Corte destaca como un propósito expreso de la Ley 50/9016

En cuanto a sentencias de revisión de tutelas, la Corte Constitucional ha estudiado diversas situaciones respecto de las EST en relación con los derechos fundamentales. El tema ha sido analizado directa o indirectamente en sentencias como las siguientes: T-739/98, T-1562/00, T-1101/01 y T-1280/01. De las sentencias de tutela mencionadas, la T-1101/01 señala en forma bastante completa la apreciación de las EST a la luz de la Constitución, así como la problemática práctica que las mismas registran.

En la sentencia T-1101/01, con ponencia del Magistrado Manuel José Cepeda ${ }^{17}$, la Corte estudió en revisión un caso de una trabajadora contratada por una EST para realizar labores en la usuaria, habiendo prestado sus servicios durante siete meses; a la trabajadora le fue terminado el contrato de trabajo «por finalización de la obra o labor», pese a que había notificado su estado de embarazo, y sin que la EST hubiera solicitado permiso al Ministerio del Trabajo para proceder al despido. Las pruebas practicadas por la Corte aportaron otros datos de interés: que la usuaria ha contratado los servicios de la temporal implicada en el caso y de otras EST para atender incrementos de producción por períodos de tiempo superiores a los autorizados por la ley; además, se estableció la precariedad de los mecanismos de vigilancia y control aplicados por el Minis220 terio a las EST. Las tesis más importantes de esta sentencia, desde el punto de vista que se viene estudiando, son las siguientes:

a) La Corte Constitucional reitera su jurisprudencia sobre la «estabilidad laboral reforzada» que tiene la trabajadora embarazada, así como la trascendencia constitucional de esa protección. Destaca la Corte que como consecuencia de ese derecho de estabilidad, «se restringe la autonomía de la voluntad contractual entre el empleador y su trabajadora», por lo cual aún la terminación del contrato por extinción del plazo fijo pactado resulta contraria a la Constitución. Recuer-

\footnotetext{
${ }^{16}$ Además de la sentencia examinada, la Corte Constitucional ha proferido las sentencias C358/99 y C-1113/01, en las cuales se demandaron normas del régimen legal de las EST. La Corte produjo en ambos casos sentencias inhibitorias por deficiencias en la argumentación constitucional de las demandas.

${ }^{17}$ Revista Tutela, Tomo III, p. 317. Legis, Bogotá, 2002.
} 
da la Corte que aunque la tutela no es el mecanismo normal para obtener reintegro laboral, en estos casos de protección a la maternidad sí procede como mecanismo transitorio de protección.

b) Destaca luego la Corte que esta protección constitucional a la maternidad procede «cualquiera que sea el tipo de contrato que da origen a la relación laboral, incluida la modalidad de contrato de servicios temporales». Luego de examinar brevemente el marco legal de las EST pasa a analizar el caso concreto y encuentra que en ese caso, no hubo los «incrementos de producción» que se tuvieron como causa de la contratación, ni que la causa de la terminación del contrato de trabajo entre la trabajadora y la EST haya sido la «finalización de la obra para la cual fue contratada», por lo cual concluyó que «la razón de la terminación del contrato con la actora fue su embarazo».

c) Posteriormente sienta la Corte un criterio importante: considera que cuando la irregularidad de la contratación de servicios temporales y la vulneración de derechos es tan evidente, «se desvirtúa el carácter de trabajo temporal y las prerrogativas de ese tipo de contratos». Por eso concedió la tutela ordenando el reintegro de la trabajadora al lugar de trabajo en la empresa usuaria «si su solicitud de trabajadores temporales sigue vigente», o en cualquiera de las empresas clientes de la EST «que desarrollen actividades en el mismo sector de producción» de la usuaria.

d) Finalmente la Corte, a partir del caso en estudio, cuestiona la realidad del trabajo a través de EST en Colombia y en particular las siguientes prácticas frecuentes e ilegales: la subrogación sin restricciones entre empresas temporales para eludir el límite legal a la contratación; la contratación de temporales para actividades que no son temporales sino necesarias y propias de la producción delas usuarias; la ausencia de efectivos controles administrativos del Ministerio de Trabajo, etc. Por esas razones, la Corte ordenó al Ministerio que en un plazo de seis meses (que ya han trascurrido), diseñe, adopte y ejecute

un programa que garantice efectivamente los derechos de los trabajadores temporales y establezca los correctivos necesarios que eviten que las empresas de servicios temporales y sus usuarias incurran en graves irregularidades para beneficiarse de las ventajas que ofrecen este tipo de contratos.

\section{Reflexiones finales}

Como puede observarse, las decisiones de la jurisprudencia acerca de la problemática del empleo a través de las EST, marcan importantes tendencias: Con la orden al Ministerio de Trabajo de fortalecer la política de vigilancia y control de esta actividad, es de esperar que en el futuro próximo haya una más eficiente actitud por parte de esa dependencia. Además, tanto la juris- 
prudencia de la Corte Suprema de Justicia como de la Corte Constitucional destacan que la actividad de las EST tiene unas importantes ventajas legales, que están condicionadas al respeto estricto al marco legal de su actividad, de modo que si hay excesos o desconocimientos de ese marco legal, desaparecen esas ventajas normativas y habrá responsabilidades directas de la empresa usuaria con los trabajadores temporales. 